\title{
Papaverine Exerts Neuroprotective Effect by Inhibiting NLRP3 Inflammasome Activation in an MPTP-Induced Microglial Priming Mouse Model Challenged with LPS
}

\author{
Yea-Hyun Leem, Jin-Sun Park, Jung-Eun Park, Do-Yeon Kim and Hee-Sun Kim* \\ Department of Molecular Medicine and the Ewha Medical Research Institute, School of Medicine, Ewha Womans University, Seoul \\ 07804, Republic of Korea
}

\begin{abstract}
Microglial priming is the process of microglial proliferation and activation in response to neurodegeneration and abnormal protein accumulation. Priming makes microglia susceptible to secondary inflammatory stimuli and causes exaggerated inflammatory responses. In the present study, we established a microglial priming model in mice by administering a single injection of 1-methyl4-phenyl-1,2,3,6-tetrahydropyridine (MPTP, $20 \mathrm{mg} / \mathrm{kg}$ ). MPTP induced microglial activation without dopaminergic degeneration; however, subsequent treatment with a sub-toxic dose of lipopolysaccharides (LPS) induced an amplified inflammatory response and caused nigrostriatal dopaminergic degeneration. These pathological and inflammatory changes, including microglial activation and dopaminergic cell loss in the substantia nigra (SN) area were reversed by papaverine (PAP) administration. In addition, MPTP/LPS enhanced interleukin-1 $\beta$ (IL-1 $\beta$ ) expression and processing via nod-like receptor protein 3 (NLRP3) inflammasome activation in the SN region of mice. However, PAP treatment suppressed inflammasome activation and subsequent IL-1 $\beta$ maturation. Moreover, PAP inhibited nuclear factor- $\kappa B(N F-\kappa B)$ and enhanced $c A M P$-response element binding protein $(C R E B)$ activity in the SN of MPTP/LPS mice. These results suggest that PAP inhibits the activation of NLRP3 inflammasome by modulating NF- $\kappa B$ and CREB signaling pathways, which results in reduced microglial activation and neuronal cell death. Thus, PAP may be a potential candidate for the treatment of Parkinsons's disease, which is aggravated by systemic inflammation.
\end{abstract}

Key Words: Parkinson's disease, Microglial priming, Systemic inflammation, Papaverine, NLRP3 inflammasome, Neuronal cell death

\section{INTRODUCTION}

Parkinson's disease (PD) is a neurodegenerative disease characterized by impaired movement, muscular rigidity, resting tremor, bradykinesia, and postural instability (Przedborski, 2017). Along with nigrostriatal dopaminergic degeneration and cytoplasmic aggregate inclusion, persistent neuroinflammation is a neuropathological feature of PD and contributes to the progression of PD (Liu et al., 2019; Pajares et al., 2020). Therefore, neuroinflammation is considered a potential therapeutic target in the treatment of PD.

Microglia are usually primed by pathological conditions, resulting in an amplified immune response to secondary inflammatory challenges (Perry and Teeling, 2013; Neher and Cunningham, 2019). The pathological significance of primed microglia both in aging and in neurodegenerative diseases has previously been studied (Perry and Holmes, 2014; Norden et al., 2015). Microglial priming has been particularly observed in PD patients and rodent models (Langston et al., 1999; Perry and Holmes, 2014). Elevated levels of pro-inflammatory cytokines such as tumor necrosis factor- $\alpha$ (TNF- $\alpha$ ), interleukin$1 \beta$ (IL-1 $)$, and IL-18 have been observed in the striatum and cerebrospinal fluid of PD patients and in the substantia nigra (SN) of PD animal models (Mogi et al., 1996; Hébert et al., 2003). Primed microglia aggravated nigrostriatal degeneration during subsequent immune challenge and further increased the release of pro-inflammatory cytokines such as IL-1 $1 \beta$ compared to non-primed cells (Pott Godoy et al., 2008; Dwyer et al., 2020). Several studies have demonstrated that IL-1 $\beta$ is a key component of the inflammatory response that leads to PD onset or exacerbation (Koprich et al., 2008; Pott Godoy et al., 2008).

The activity of IL-1 $\beta$ is particularly controlled by a cytosolic multimolecular complex termed 'inflammasome,' which con-

\section{Open Access https://doi.org/10.4062/biomolther.2021.039}

This is an Open Access article distributed under the terms of the Creative Commons Attribution Non-Commercial License (http://creativecommons.org/licenses/by-nc/4.0/) which permits unrestricted non-commercial use, distribution, and reproduction in any medium, provided the original work is properly cited.
Received Feb 26, 2021 Revised Mar 23, 2021 Accepted Mar 26, 2021

Published Online May 1, 2021

*Corresponding Author

E-mail: hskimp@ewha.ac.kr

Tel: +82-2-6986-6270, Fax: +82-2-6986-7014 
tains nod-like receptor protein 3 (NLRP3), adaptor protein ASC, and pro-caspase-1 (Martinon et al., 2002; Seok et al., 2021). The inflammasome proceeds in two steps: the priming step first upregulates the expression of inflammasome components, including NLRP3 and pro-IL-1 $\beta / I L-18$, and the activation step then induces caspase-1-dependent proteolytic maturation of IL-1 $\beta$ and IL-18 (Swanson et al., 2019). NLRP3, caspase- 1 , and IL-1 $\beta$ levels are found to be elevated in PD patients and several studies have reported the role of inflammasomes in PD pathogenesis (Mogi et al., 2000; von Herrmann et al., 2018). Martinez et al. (2017) reported that NLRP3 causes inflammatory changes and nigral cell loss in rotenoneexposed mice. The dopaminergic degeneration in 1-methyl4-phenyl-1,2,3,6-tetrahydropyridine (MPTP)-treated mice was rescued by suppressing inflammasomes, wherein the chemical inhibition or genetic deletion of NLRP3 mitigated neuroinflammation and inhibited $\alpha$-synuclein aggregation (Gordon et al., 2018; Lee et al., 2019a). Wang et al. (2016) reported that caspase- 1 induces truncation and aggregation of $\alpha$-synuclein, which is a PD-associated protein. Thus, it is essential to develop therapeutic agents that can control NLRP3 inflammasome and the subsequent IL-1 $\beta$ processing to prevent neuroinflammation and dopaminergic neuronal cell death.

In the present study, we examined the effect of papaverine (PAP; 6,7-dimethoxy-1-veratryl-isoquinoline) in an MPTPinduced microglial priming mouse model challenged with lipopolysaccharide (LPS). PAP is a non-narcotic opium alkaloid isolated from Papaver somniferum, and is clinically used to treat conditions associated with spasm and dysmotility of the gastrointestinal tract (Han et al., 2010; Zhu et al., 2014). PAP is a selective inhibitor of phosphodiesterase 10A (PDE10A) that is highly expressed in the striatum, $\mathrm{SN}$, and olfactory tubercle (Wilson and Brandon, 2015). In this study, we demonstrated that PAP inhibits microglial activation and dopaminergic neuronal cell death by modulating NLRP3 inflammasome activation in MPTP/LPS mice; this indicates the therapeutic potential of PAP in PD, which is exacerbated by systemic inflammation.

\section{MATERIALS AND METHODS}

\section{Animals}

Adult male C57BL/6 mice (9 weeks of age) were purchased from Orient Bio Inc. (Seongnam, Korea), a branch of Charles River Laboratories. Mice were maintained at $21^{\circ} \mathrm{C}$ under a 12 $\mathrm{h}$ light: $12 \mathrm{~h}$ dark cycle and had ad libitum access to water and rodent chow. Every effort was made to minimize animal suffering. All experiments were performed in accordance with the National Institutes of Health (NIH, Bethesda, MD, USA) and Ewha Womans University (Seoul, Korea) guidelines for laboratory animal care and use, and the study was approved by the Institutional Animal Care and Use Committee of the Medical School of Ewha Womans University (\#EUM 20-022).

\section{MPTP-induced microglia priming and drug administration}

Mice were randomly divided into the following five groups ( $N=10-16)$ : 1. CON (control); 2. MPTP; 3. LPS; 4. MPTP/LPS, MPTP+LPS; 5. MPTP/LPS+PAP, MPTP+LPS+papaverine. Mice were administered MPTP (20 mg/kg, i.p.), followed by 3 consecutive days of PAP administration ( $20 \mathrm{mg} / \mathrm{kg}$, i.p.). Mice were administered LPS $(0.5 \mathrm{mg} / \mathrm{kg}$, i.p.) $1 \mathrm{~d}$ after the last treatment with PAP and were sacrificed 3 days after LPS administration. LPS and PAP were obtained from Sigma-Aldrich (St. Louis, MO, USA). MPTP was purchased from Tokyo Chemical Industry Co (Tokyo, Japan). A schematic of this procedure is shown in Fig. $1 \mathrm{~A}$.

\section{Histological analysis}

Mice were anesthetized with sodium pentobarbital $(80 \mathrm{mg} /$ kg; Hanlim Pharm Co. Ltd., Seoul, Korea) to induce rapid and prolonged anesthesia. The mice were then perfused transcardially, their brains were removed, and $40 \mu \mathrm{m}$-thick sections were prepared using a cryotome. For immunohistochemistry (IHC) analysis, the sections were subjected to endogenous peroxidation inactivation with $3 \%$ hydrogen peroxide $\left(\mathrm{H}_{2} \mathrm{O}_{2}\right)$, and non-specific binding was blocked with $4 \%$ bovine serum albumin (BSA). The sections were first incubated overnight
A

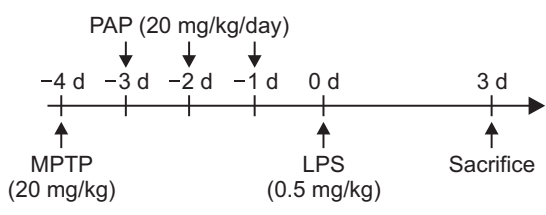

C

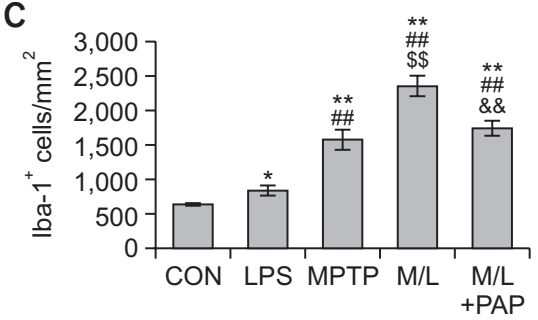

\section{B}

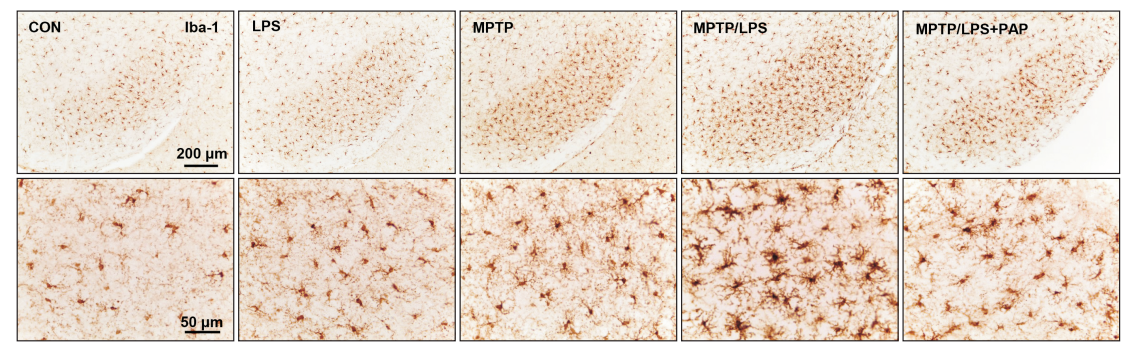

Fig. 1. PAP attenuated hyperreactive microglia in the brains of MPTP/LPS-treated mice. (A) The experimental procedure: Mice were subjected to a single injection of MPTP $(20 \mathrm{mg} / \mathrm{kg})$, followed by administration of PAP (20 mg/kg/day) for three days. The mice were then treated with a low dose of LPS (i.p., $0.5 \mathrm{mg} / \mathrm{kg}$ ), and sacrificed three days later. (B) Photomicrographs of the immunoreactivity of SN Iba- $1^{+}$cells ( $N=5-8$ in each group). (C) Quantification of the immunoreactivity of SN Iba- $1^{+}$cells. Statisctical analyses were performed for all data using ANOVA with post-hoc test of the LSD. Data are presented as mean \pm SEM. ${ }^{*} p<0.05$, vs. CON; ${ }^{* *} p<0.01$, vs. CON; ${ }^{\#} p<0.01$, vs. LPS; ${ }^{\$ \$} p<0.01$, vs. MPTP; ${ }^{\& \&} p<0.01$, vs. M/L. CON, control; M/L, MPTP/LPS; M/L+PAP, MPTP/LPS+PAP. 
with primary antibodies and were then incubated with biotinylated secondary antibodies for $1 \mathrm{~h}$ at $25^{\circ} \mathrm{C}$, on the following day. The sections were subsequently incubated with avidinbiotin-horse radish peroxidase (HRP) complex reagent solution for $1.5 \mathrm{~h}$ (Vector Laboratories, Burlingame, CA, USA) and a peroxidase reaction was then performed using diaminobenzidine tetrahydrochloride (Vector Laboratories). For double immunofluorescence (IF) analysis, non-specific binding was blocked, and the sections were incubated with primary antibodies followed by fluorochrome-conjugated secondary antibodies. The tissue was then mounted with an antifade reagent (Vector Laboratories). Digital images of IHC and IF staining were captured using a Leica DM750 microscope (Leica Microsystems, Nussloch, Germany). Quantification was performed using ImageJ (NIH Image Engineering, Bethesda, MD, USA) and AxioVision (Carl Zeiss Microscopy GmbH, Jena, Germany). The following primary antibodies were used in this study: anti-tyrosine hydroxylase (anti-TH), anti-IL-1 $\beta$, antiNLRP3, anti-ASC antibodies from Cell Signaling Technology, Inc. (Danvers, MA, USA), anti-ionized calcium-binding adaptor molecule 1 (anti-lba1) from Wako (Osaka, Japan), and biotinylated and fluorophore-conjugated secondary antibodies from Vector Laboratories. Anti-CD11b (OX42) was obtained from Bio-Rad (Hercules, CA, USA).

\section{Preparation of protein extracts and western blot analysis}

Tissues collected from SN were homogenized in ice-cold radioimmuno precipitation assay (RIPA) lysis buffer (10 mM Tris-Cl [pH 7.4], $300 \mathrm{mM} \mathrm{NaCl}, 1 \%$ Triton $\mathrm{X}-100,0.1 \%$ sodium dodecyl sulfate [SDS], $0.1 \%$ sodium deoxycholate, $1 \mathrm{mM}$ ethylenediaminetetraacetic acid) containing a protease inhibitor cocktail (Complete Mini, Roche, Mannheim, Germany). Subsequently, the samples were vortexed vigorously and incubated for $30 \mathrm{~min}$ at $4^{\circ} \mathrm{C}$. The samples were then centrifuged at $20,000 \times \mathrm{g}$ for $30 \mathrm{~min}$, and the supernatant was collected. Protein samples $(30-50 \mu \mathrm{g})$ were separated by SDS-PAGE, transferred to a nitrocellulose membrane, and incubated with primary antibodies according to the manufacturer' $s$ instructions for dilution. The membranes were then thoroughly washed with TBST (tris-buffered saline and tween 20) and incubated with HRP-conjugated secondary antibodies (BioRad; 1:3,000 dilution in TBST). Subsequently, the blots were developed using an enhanced chemiluminescence detection kit (Thermo Fisher Scientific, Waltham, MA, USA). For quantification, the density of specific target bands was normalized against $\beta$-actin using ImageJ software, version $1.37(\mathrm{NIH})$. The following primary antibodies were used in this study: antiIL-1 $\beta$, anti-caspase-1, anti-NLRP3, anti-IkB, anti-p-IkB, antiCREB, and anti-p-CREB from Cell Signaling Technology, Inc. Antibody against $\beta$-actin was purchased from Sigma-Aldrich.

\section{Statistical analysis}

Statistical analyses were performed using SPSS for Windows (version 18.0; SPSS Inc., Chicago, IL, USA). The differences among the groups were analyzed using a one-way analysis of variance (ANOVA). Post-hoc comparisons were conducted using the least significant difference (LSD) test. All values are presented as the mean \pm standard error of the mean (SEM). A value of $p<0.05$ was considered statistically significant.

\section{RESULTS}

PAP attenuated hyperreactive microglia in the SN of mice cotreated with MPTP and LPS

We first established an in vivo microglia priming model, by administering a single injection of MPTP $(20 \mathrm{mg} / \mathrm{kg})$ followed by a sub-toxic dose of LPS $(0.5 \mathrm{mg} / \mathrm{kg})(\mathrm{Fig} .1 \mathrm{~A})$. The number of $\mathrm{Iba}-1^{+}$cells in the MPTP (primed/pre-activated microglia) or LPS (the second inflammatory stimulator) group were significantly higher than the CON group, and $\mathrm{Iba}-1^{+}$cells were significantly enhanced in the MPTP group compared to the LPS group. The number of Iba- $1^{+}$cells in the SN of the MPTP/ LPS group (hyperreactive microglia) increased substantially compared to the LPS and MPTP group, while PAP significantly reduced Iba- $1^{+}$cells (Fig. $1 \mathrm{~B}, 1 \mathrm{C} ; \mathrm{F}_{4,26}=33.23, p<0.01$ ). Cytoarchitecture revealed increased hypertrophy of microglial processes and soma in the MPTP/LPS group than in the LPS or MPTP groups, which in turn was reversed by PAP (Fig. 1B). The microglial morphology of the LPS group was similar to that of the CON group. This result indicated that the phenotype of MPTP-triggered primed microglia shifted from pre-activated (mildly reactive) to hyperreactive following LPS treatment, while PAP treatment alleviated the exaggerated inflammatory response. Therefore, our experimental paradigm was relevant for exploring the effects of PAP in a primed microglia model.

\section{PAP alleviated nigrostriatal dopaminergic degeneration in mice cotreated with MPTP and LPS}

When we examined the effect of MPTP or LPS treatment on the survival of dopaminergic neurons, the number of $\mathrm{TH}^{+}$ dopaminergic cells did not change significantly compared with the control group (Fig. 2). However, the number of $\mathrm{TH}^{+}$cells in the SN of the MPTP/LPS group was profoundly reduced compared to the other groups, including the CON, LPS, and MPTP groups; cell death was then recovered by PAP treatment (Fig. $\left.2 \mathrm{~A}, 2 \mathrm{~B} ; \mathrm{F}_{4,27}=8.362, p<0.01\right)$. In addition, striatal $\mathrm{TH}^{+}$immunoreactivity corresponded well with the results from SN (Fig. $\left.2 \mathrm{~A}, 2 \mathrm{C} ; \mathrm{F}_{4,27}=9.09, p<0.01\right)$. The results indicated that primed/ pre-activated microglia induced only by MPTP or the second inflammatory stimulus (LPS) did not produce nigrostriatal dopaminergic degeneration, whereas hyperreactive microglia caused nigrostriatal dopaminergic degeneration in MPTP/LPS mice. In addition, PAP probably has an ameliorating effect on dopaminergic degeneration.

\section{PAP reduced microglial IL-1 $\beta$ expression in the SN of mice cotreated with MPTP and LPS}

Next, we examined the effect of MPTP and LPS on the expression of IL-1 $\beta$, which plays a major role in microglial priming and the exacerbation of neuronal loss (Koprich et al., 2008; Pott Godoy et al., 2008). We found that the IL-1 $\beta^{+}$immunoreactivities of the LPS and MPTP groups were significantly enhanced compared to the CON group, and the IL- $1 \beta^{+}$fluorescence intensities of the MPTP/LPS group were higher than the LPS or MPTP group (Fig. 3A, 3B; $F_{4,15}=20.97, p<0.01$ ). Moreover, the area of IL-1 $\beta$ that overlapped with OX42-positive microglial cells significantly increased in the MPTP/LPS group compared to the LPS or MPTP group (Fig. 3A, 3C; $F_{4,15}=17.54, p<0.01$ ). This result suggests that hyperreactive microglia produce a multiplied inflammatory response to the subsequent LPS treatment, thereby amplifying IL-1 $\beta$ protein expression in our experimental paradigm. In addition, PAP treatment significantly reduced IL- 
A

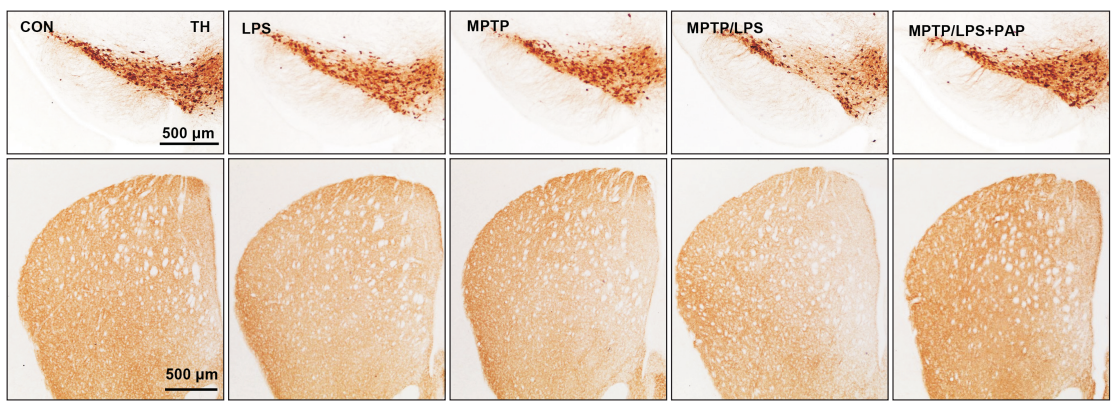

B

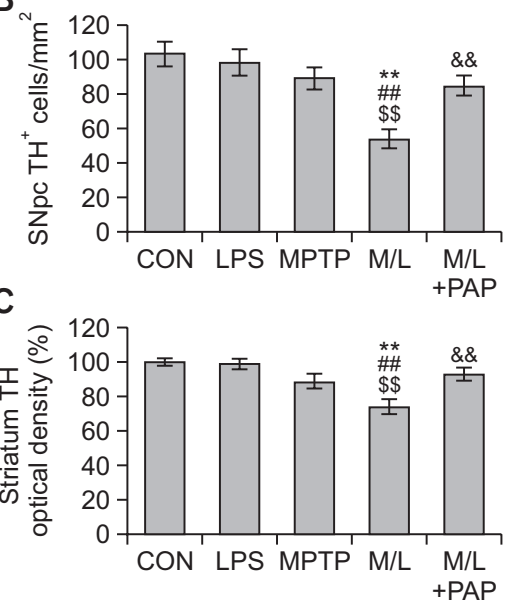

Fig. 2. PAP alleviated nigrostriatal dopaminergic degeneration in MPTP/LPS-treated mice. (A) Photomicrographs of the immunoreactivity of $\mathrm{SN}$ and striatal $\mathrm{TH}^{+}$cells $\left(\mathrm{N}=6-8\right.$ in each group). (B, C) Quantification of the immunoreactivity of $\mathrm{SN}$ and striatal TH $\mathrm{T}^{+}$cells. Data are presented as mean \pm SEM. ${ }^{* *} p<0.01$, vs. CON; ${ }^{\# \#} p<0.01$, vs. LPS; ${ }^{\$ \$} p<0.01$, vs. MPTP; ${ }^{2 \&} p<0.01$, vs. M/L. TH, tyroxine hydroxylase; SNpc, Substantia nigra par compacta.

A

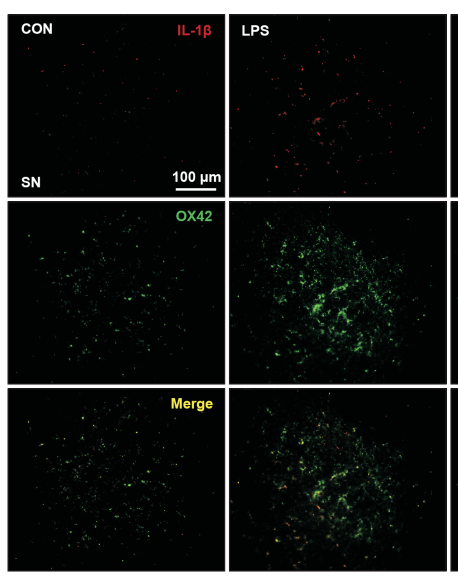

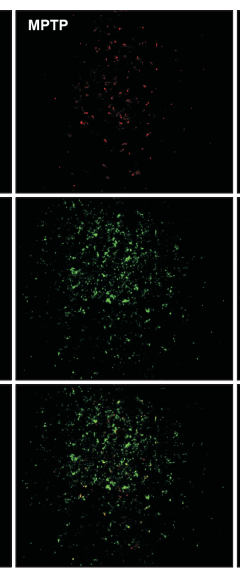

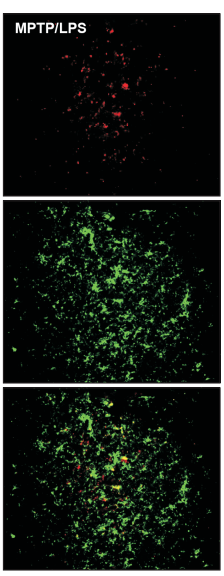

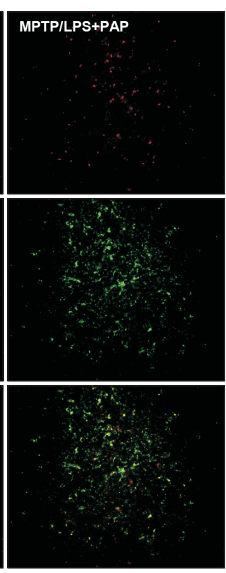

B

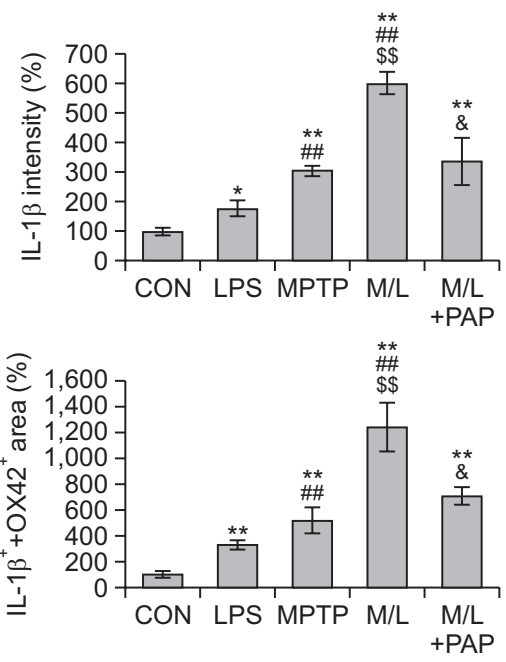

Fig. 3. PAP reduced IL-1 $\beta$ expression in microglia of MPTP/LPS-treated mice. (A) Photomicrographs of the double fluorescent staining of SN OX $42^{+}$and IL-1 $\beta^{+}$Particles (N=4 in each group). (B) Quantification of the fluorescent intensity of SN IL-1 $\beta^{+}$particles. (C) Quantification of the area of SN OX $42^{+}$and IL- $1 \beta^{+}$particles. Data are presented as mean \pm SEM. ${ }^{*} p<0.05$, vs. CON; ${ }^{* *} p<0.01$, vs. CON; ${ }^{\# \#} p<0.01$, vs. LPS; ${ }^{\$ \$} p<0.01$, vs. MPTP; ${ }^{2} p<0.05$, vs. M/L

$1 \beta$ expression in the MPTP/LPS group, which corroborates the anti-inflammatory effect of PAP.

\section{PAP inhibited microglial NLRP3 expression in the SN of mice cotreated with MPTP and LPS}

The changes in IL-1 $\beta$ expression prompted us to measure the expression of microglial NLRP3 to identify the involvement of inflammasomes. We found that NLRP3 ${ }^{+}$fluorescence intensities of the MPTP/LPS group were considerably augmented compared to the LPS or MPTP group, which was then reversed by PAP treatment (Fig. 4A-4C; $F_{4,15}=26.75, p<0.01$ ). In addition, the area of NLRP3 ${ }^{+}$that was merged with $\mathrm{OX} 42^{+}$ fluorescent signal, corresponded to the NLRP3 expression data (Fig. 4A, 4D; $F_{4,15}=46.11, p<0.01$ ). This result indicated that hyperreactive microglia strongly induced NLRP3 protein expression, whereas PAP dampened microglial NLRP3 expression.

\section{PAP reduced the ASC specks in SN of mice cotreated with MPTP and LPS}

The number of ASC specks, a hallmark of inflammasome activation, in the LPS and MPTP group increased significantly compared to the CON group, while ASC specks of the MPTP/ LPS group were markedly enhanced relative to the LPS or MPTP group (Fig. 5A-5C; $F_{4,23}=13.21, p<0.01$ ). Moreover, ASC specks in the MPTP/LPS group were reduced by PAP treatment. This result indicates that hyperreactive microglia accelerate inflammasome activation during the subsequent in- 
A

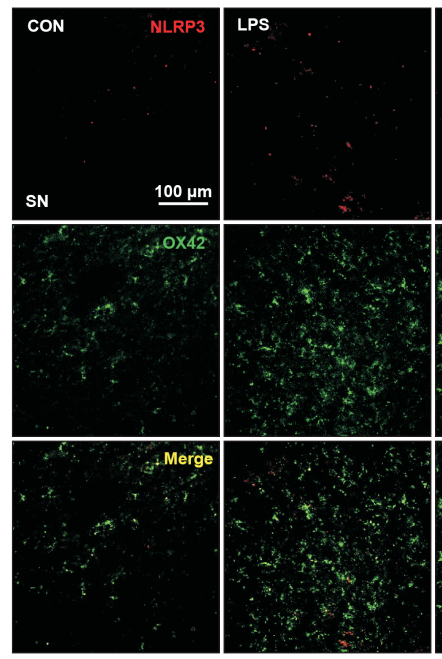

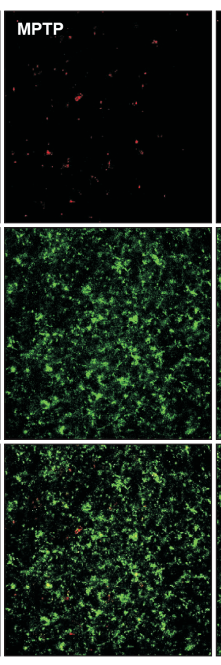

B

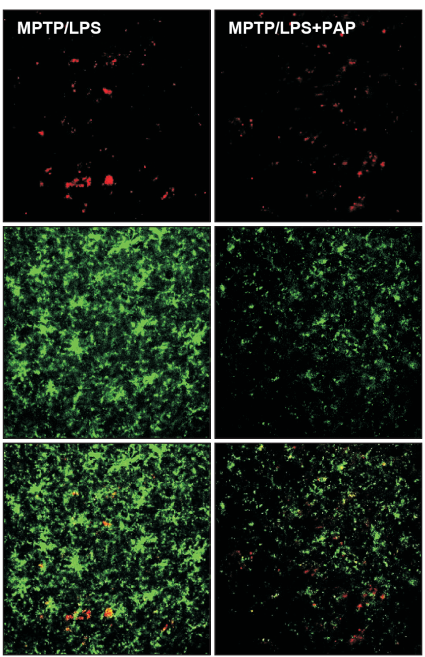

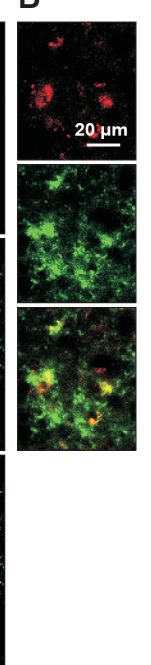

C
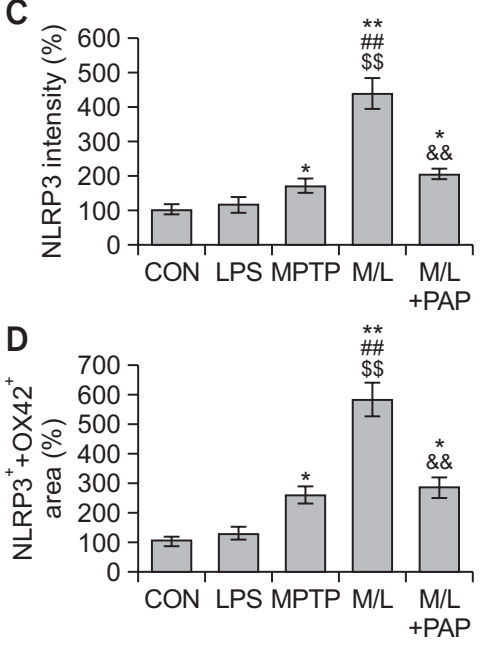

Fig. 4. PAP inhibited NLRP3 expression in microglia of MPTP/LPS-treated mice. (A) Photomicrographs of the double fluorescent staining of $\mathrm{SN} \mathrm{OX} 42^{+}$and NLRP3 ${ }^{+}$particles (N=4 in each group). (B) The high-magnitude of image. (C) Quantification of the fluorescent intensity of SN NLRP3 ${ }^{+}$particles. (D) Quantification of the area of SN OX42 ${ }^{+}$and NLRP3 ${ }^{+}$particles. Data are presented as mean $\pm \mathrm{SEM}$. ${ }^{*} p<0.05$, vs. CON; ${ }^{* *} p<0.01$, vs. CON; ${ }^{\#} p<0.01$, vs. LPS; ${ }^{\$ \$} p<0.01$, vs. MPTP; ${ }^{2 \&} p<0.01$, vs. M/L.

A

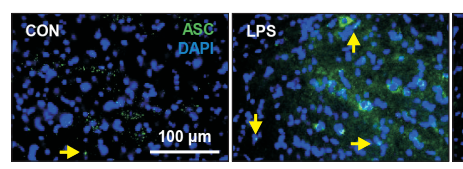

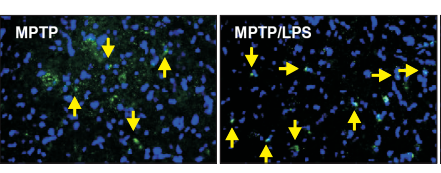

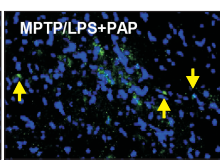

B

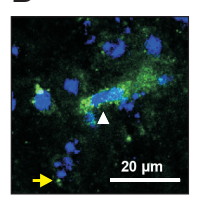

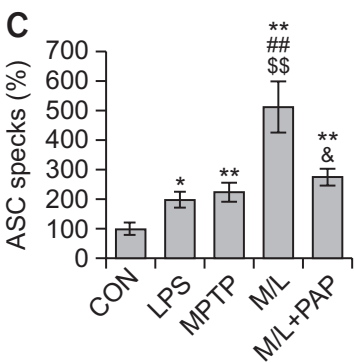

Fig. 5. PAP reduced ASC specks in MPTP/LPS-treated mice. (A) Photomicrographs of the double fluorescent staining of $S N A S C^{+}$and DAPI ${ }^{+}$particles ( $\mathrm{N}=5-7$ in each group). (B) The high-magnitude of image. In non-assembly, ASC is localized to the cytoplasm and nucleus of the cell (white arrow head). During inflammasome activation, one large ASC speck (around $1 \mu \mathrm{m}$ in diameter) assembles in the perinuclear area of the cell (yellow arrow). (C) Quantification of the number of SN ASC specks. Data are presented as mean \pm SEM. * $p<0.05$, vs. CON; ${ }^{* *} p<0.01$, vs. CON; ${ }^{\#} p<0.01$, vs. LPS; ${ }^{\$} p<0.01$, vs. MPTP; ${ }^{\star} p<0.05$, vs. M/L.

flammatory stimuli, while PAP has an inflammasome-inhibiting effect.

\section{PAP suppressed the expression and activation of NLRP3 inflammasome by modulating NF-KB/CREB signaling in the SN of mice cotreated with MPTP and LPS}

To further investigate the mechanism underlying inflammasome inhibition by PAP, we performed western blot analysis to determine the protein expression levels of NLRP3 inflammasome components and its related signaling molecules such as $\mathrm{NF}-\mathrm{kB}$ and CREB. The results showed that total IL-1 $\beta$ protein levels (pro- and mature IL-1 $\beta$ ) in the LPS or MPTP group increased significantly compared to the CON group. The cytokine levels in the MPTP/LPS group were higher than in the LPS or MPTP group, which was reversed by PAP treatment (Fig. $6 \mathrm{~A}$, $\left.6 \mathrm{~B} ; \mathrm{F}_{4,15}=25.21, p<0.01\right)$. In accordance with this, mature IL$1 \beta$ levels in the MPTP/LPS group were significantly augmented relative to the other groups, which was however significantly attenuated by PAP (Fig. $6 A$, $6 B$; $F_{4,5}=8.12, p<0.01$ ). Mature IL-
$1 \beta$ levels in the LPS and MPTP groups were comparable to those in the CON group. PAP significantly inhibited the NLRP3 protein expression induced by MPTP/LPS, while the levels of NLRP3 in the LPS and MPTP groups were similar to those in the CON group (Fig. 6A, 6B; $F_{4,15}=10.87, p<0.01$ ). Caspase-1 activity (cleaved/pro-caspase-1) was profoundly enhanced in the MPTP/LPS group compared to the LPS or MPTP group, which was reversed by PAP (Fig. 6A, 6B; $F_{4,15}=38.52, p<0.01$ ).

With regard to the signaling pathway, MPTP/LPS significantly increased NF-kB activity ( $p$-lkB/total IkB), which was inhibited by PAP. However, there was no significant difference among the CON, LPS, and MPTP groups (Fig. 6C, 6D; $\left.F_{4,15}=22.58, p<0.01\right)$. Conversely, single or combined treatment with MPTP and LPS significantly increased CREB activity ( $p-C R E B /$ total $C R E B$ ), which was augmented by PAP (Fig. 6C, 6D; $F_{4,15}=22.58, p<0.01$ ). These data imply that PAP suppressed IL-1 $\beta$ expression and inflammasome-dependent $\mathrm{IL}-1 \beta$ maturation by inhibiting NF- $\kappa \mathrm{B}$ and upregulating CREB signaling in MPTP/LPS-treated mice. 


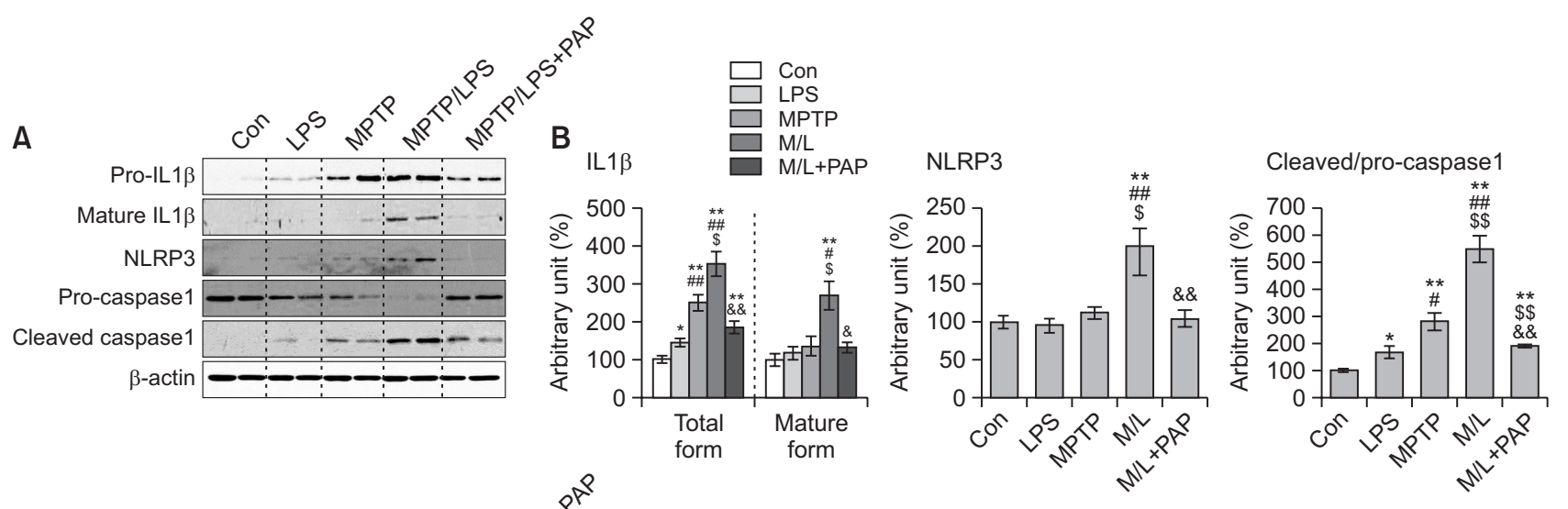

C

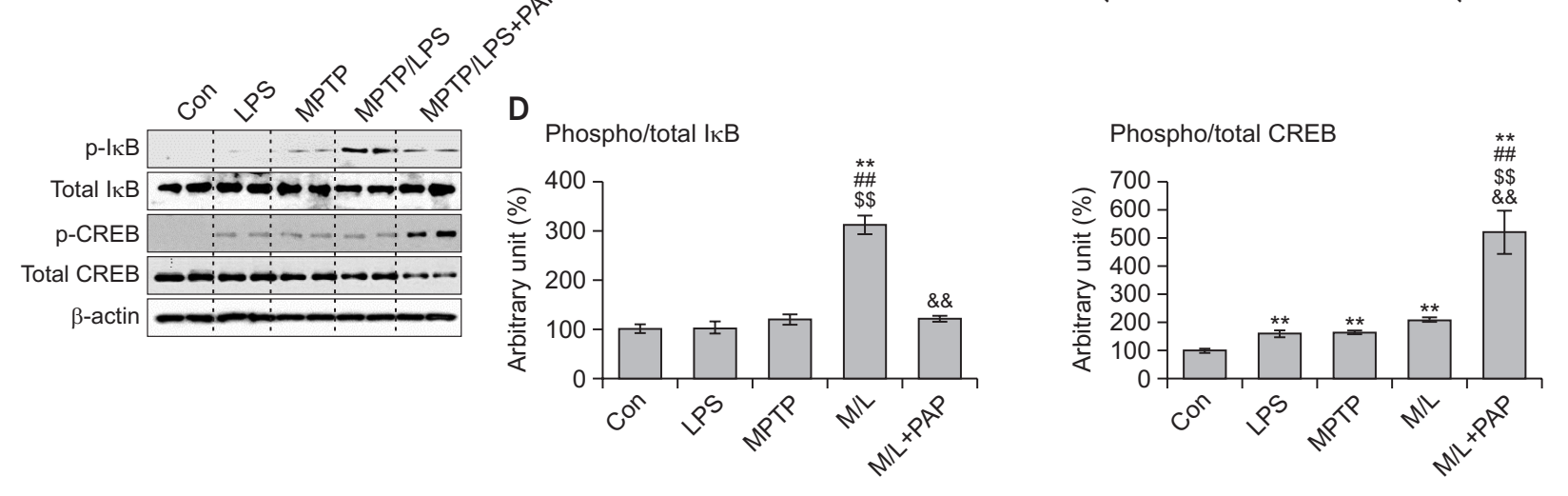

Fig. 6. Effects of PAP on the expression of NLRP3 inflammasome components and the activities of NF- $\mathrm{B}$ and CREB. (A, B) Photomicrographs (A) and quantification (B) of western blot analysis of the protein expression of IL-1 $\beta, N L R P 3$, and caspase-1 in the $S N(N=4$ in each group). (C, D) Photomicrographs (C) and quantification (D) of western blot analysis of protein expression of $p$-lkB, IkB, p-CREB, and CREB in the SN (N=4 in each group). Data are presented as mean \pm SEM. ${ }^{*} p<0.05$, vs. CON; ${ }^{* *} p<0.01$, vs. CON; ${ }^{*} p<0.05$, vs. LPS; ${ }^{* \#} p<0.01$, vs. LPS; ${ }^{\$} p<0.05$, vs. MPTP; ${ }^{\$ \$} p<0.01$, vs. MPTP; ${ }^{\&} p<0.05$, vs. M/L; ${ }^{8 \&} p<0.01$, vs. M/L.

\section{DISCUSSION}

In the current study, we demonstrated that MPTP-induced microglial priming exacerbated nigrostriatal dopaminergic degeneration and amplified the inflammatory response to subsequent systemic inflammation induced by a sub-toxic dose of LPS. The priming effects of MPTP were alleviated by the administration of PAP. Thus, PAP inhibited microglial activation and dopaminergic neuronal cell death in MPTP/LPS-treated mice. PAP administration particularly suppressed NLRP3 inflammasome-dependent IL-1 $\beta$ processing, which potentially contributes to the anti-inflammatory and neuroprotective effects of PAP.

PAP, which is a selective PDE10 inhibitor, inhibits phosphodiesterase activity and increases the intracellular CAMP level (Zagorska et al., 2018). Previous studies have reported that cAMP negatively regulates NLRP3 inflammasome activation (Lee et al., 2012; Yan et al., 2015). Yan et al. (2015) demonstrated that CAMP binds to NLRP3 and subsequently induces NLRP3 ubiquitination/degradation in bone marrow macrophages and in mouse models of MPTP-induced neuroinflammation, LPS-induced systemic inflammation, and MSU-induced peritoneal inflammation. Consistent with these studies, we showed that PAP inhibited NLRP3 protein levels in MPTP/ LPS-induced neuroinflammation in mice. This effect is likely to be associated with PAP-induced increase in cAMP levels.
Corroborating this, our group recently reported that PAP increased the intracellular level of cAMP in LPS-stimulated microglia (Lee et al., 2019b). Moreover, we demonstrated that PAP inhibited microglial activation and dopaminergic neuronal cell death via PKA/CREB signaling.

Another mechanism underlying the inhibition of NLRP3 inflammasome by PAP could possibly be NF-kB inhibition. In response to activation of receptors such as toll-like receptors (TLR2/4) and TNF receptors by priming signals, NF-KB is activated and upregulates the expression of inflammasome components such as NLRP3 and pro-IL1 $\beta / I L-18$ (Seok et al., 2021). In the present study, we found that PAP inhibited NF-kB activity, resulting in reduced expression of NLRP3 and IL-1 $\beta$.

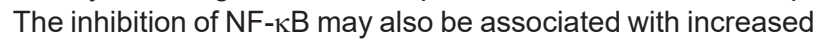
CREB signaling, because $\mathrm{p}-\mathrm{CREB}$ is known to inhibit NF-kBmediated transcription by competing for limited amounts of coactivator CREB binding protein (CBP) (Wen et al., 2010). Taken together, the present study suggests two possible mechanisms of PAP in MPTP/LPS-treated mouse brains. First, PAP may suppress microglial inflammasome activation by promoting CAMP-mediated NLRP3 degradation. Second, PAP-mediated inhibition of NF- $\mathrm{NB}$ activity may block inflammasome activation by downregulating the synthesis of its components. The inhibition of NF-kB may also be associated with CREB phosphorylation induced by PAP. Further studies are necessary to clarify the mechanism underlying inflamma- 
some inhibition by PAP.

Previous studies have reported the therapeutic effects of PAP in various neuropathological conditions. PAP improved cognitive impairment in a Huntington's disease mouse model by increasing GluA1 and CREB phosphorylation (Giralt et al., 2013). PAP is a potent PDE10 inhibitor that activates cAMP/ PKA signaling in striatopallidal and striatonigral medium spiny neurons, which indicates the therapeutic potential of PAP in neuropsychotic disorders such as schizophrenia (Zagorska et al., 2018). Recently, our group reported the therapeutic effects of PAP in PD mouse models (Lee et al., 2019b; Leem et al., 2020). PAP exerts neuroprotective and anti-inflammatory effects in an MPTP-induced PD mouse model (Lee et al., 2019b). In addition, PAP inhibits $\alpha$-synuclein aggregation by modulating neuroinflammation and matrix metalloproteinase-3 expression in the subacute MPTP/probenecid mouse model of PD (Leem et al., 2020).

Our study reports for the first time the therapeutic effect of PAP in an MPTP-induced microglial priming model challenged with LPS, and the involvement of NLRP3 inflammasome in this mechanism. Considering that PAP is a non-addictive opium alkaloid with few side effects, it is a potential therapeutic candidate for PD and other neurodegenerative diseases that are associated with microglial priming and activation.

\section{CONFLICT OF INTEREST}

The authors have no conflict of interest to declare.

\section{ACKNOWLEDGMENTS}

This research was supported by the National Research Foundation of Korea (NRF) grant funded by the Korea government (MSIT) (2016R1A6A3A11930120, 2018R1A2B6003074 and 2020R1I1A1A01057922).

\section{REFERENCES}

Dwyer, Z., Rudyk, C., Thompson, A., Farmer, K., Fenner, B., Fortin, T., Derksen, A., Sun, H. and Hayley, S.; CLINT (Canadian LRRK2 in inflammation team) (2020) Leucine-rich repeat kinase-2 (LRRK2) modulates microglial phenotype and dopaminergic neurodegeneration. Neurobiol. Aging 91, 45-55.

Giralt, A., Saavedra, A., Carretón, O., Arumí, H., Tyebji, S., Alberch, J. and Pérez-Navarro, E. (2013) PDE10 inhibition increases GluA1 and CREB phosphorylation and improves spatial and recognition memories in a Huntington's disease mouse model. Hippocampus 23, 684-695.

Gordon, R., Albornoz, E. A., Christie, D. C., Langley, M. R., Kumar, V., Mantovani, S., Robertson, A. A. B., Butler, M. S., Rowe, D. B., O'Neill, L. A., Kanthasamy, A. G., Schroder, K., Cooper, M. A. and Woodruff, T. M. (2018) Inflammasome inhibition prevents alpha-synuclein pathology and dopaminergic neurodegeneration in mice. Sci. Transl. Med. 10, eaah4066.

Han, X., Lamshöft, M., Grobe, N., Ren, X., Fist, A. J., Kutchan, T. M., Spiteller, M. and Zenk, M. H. (2010) The biosynthesis of papaverine proceeds via (S)-reticuline. Phytochemistry 71, 1305-1312.

Hébert, G., Arsaut, J., Dantzer, R. and Demotes-Mainard, J. (2003) Time-course of the expression of inflammatory cytokines and matrix metalloproteinases in the striatum and mesencephalon of mice injected with 1-methyl-4-phenyl-1,2,3,6-tetrahydropyridine, a dopaminergic neurotoxin. Neurosci. Lett. 349, 191-195. von Herrmann, K. M., Salas, L. A., Martinez, E. M., Young, A. L., Howard, J. M., Feldman, M. S., Christensen, B. C., Wilkins, O. M., Lee, S. L., Hickey, W. F. and Havrda, M. C. (2018) NLRP3 expression in mesencephalic neurons and characterization of a rare NLRP3 polymorphism associated with decreased risk of Parkinson's disease. NPJ Parkinsons Dis. 4, 24

Koprich, J. B., Reske-Nielsen, C., Mithal, P. and Isacson, O. (2008) Neuroinflammation mediated by IL-1 beta increases susceptibility of dopamine neurons to degeneration in an animal model of Parkinson's disease. J. Neuroinflammation 5, 8 .

Langston, J. W., Forno, L. S., Tetrud, J., Reeves, A. G., Kaplan, J. A. and Karluk, D. (1999) Evidence of active nerve cell degeneration in the substantia nigra of humans years after 1-methyl-4-phenyl1,2,3,6-tetrahydropyridine exposure. Ann. Neurol. 46, 598-605.

Lee, E., Hwang, I., Park, S., Hong, S., Hwang, B., Cho, Y., Son, J. and Yu, J. W. (2019a) MPTP-driven NLRP3 inflammasome activation in microglia plays a central role in dopaminergic neurodegeneration. Cell Death Differ. 26, 213-228.

Lee, G. S., Subramanian, N., Kim, A. I., Aksentijevich, I., GoldbachMansky, R., Sacks, D. B., Germain, R. N., Kastner, D. L. and Chae, J. J. (2012) The calcium-sensing receptor regulates the NLRP3 inflammasome through $\mathrm{Ca}^{2+}$ and cAMP. Nature 492, 123-127.

Lee, Y. Y., Park, J. S., Leem, Y. H., Park, J. E., Kim, D. Y., Choi, Y. H., Park, E. M., Kang, J. L. and Kim, H. S. (2019b) The phosphodiesterase 10 inhibitor papaverine exerts anti-inflammatory and neuroprotective effects via the PKA signaling pathway in neuroinflammation and Parkinson's disease mouse models. J. Neuroinflammation 16, 246.

Leem, Y. H., Park, J. S., Park, J. E., Kim, D. Y., Kang, J. L. and Kim, H. S. (2020) Papaverine inhibits $\alpha$-synuclein aggregation by modulating neuroinflammation and matrix metalloproteinase-3 expression in the subacute MPTP/P mouse model of Parkinson's disease. Biomed. Pharmacother. 130, 110576.

Liu, C. Y., Wang, X., Liu, C. and Zhang, H. L. (2019) Pharmacological targeting of microglial activation: new therapeutic approach. Front. Cell. Neurosci. 13, 514.

Martinez, E. M., Young, A. L., Patankar, Y. R., Berwin, B. L., Wang, L., von Herrmann, K. M., Weier, J. M. and Havrda, M. C. (2017) Editor's highlight: NIrp3 is required for inflammatory changes and nigral cell loss resulting from chronic intragastric rotenone exposure in mice. Toxicol. Sci. 159, 64-75.

Martinon, F., Burns, K. and Tschopp, J. (2002) The inflammasome: a molecular platform triggering activation of inflammatory caspases and processing of prolL-beta. Mol. Cell 10, 417-426.

Mogi, M., Harada, M., Narabayashi, H., Inagaki, H., Minami, M. and Nagatsu, T. (1996) Interleukin (IL)-1 beta, IL-2, IL-4, IL-6 and transforming growth factor-alpha levels are elevated in ventricular cerebrospinal fluid in juvenile parkinsonism and Parkinson's disease. Neurosci. Lett. 211, 13-16.

Mogi, M., Togari, A., Kondo, T., Mizuno, Y., Komure, O., Kuno, S., Ichinose, H. and Nagatsu, T. (2000) Caspase activities and tumor necrosis factor receptor R1 (p55) level are elevated in the substantia nigra from parkinsonian brain. J. Neural Transm. (Vienna) 107, 335-341.

Neher, J. J. and Cunningham, C. (2019) Priming microglia for innate immune memory in the brain. Trends Immunol. 40, 358-374.

Norden, D. M., Muccigrosso, M. M. and Godbout, J. P. (2015) Microglial priming and enhanced reactivity to secondary insult in aging, and traumatic CNS injury, and neurodegenerative disease. Neuropharmacology 96, 29-41.

Pajares, M., Rojo, A. I., Manda, G., Bosca, L. and Cuadrado, A. (2020) Inflammation in Parkinson's disease: mechanisms and therapeutic implications. Cells 9, 1687.

Perry, V. H. and Holmes, C. (2014) Microglial priming in neurodegenerative disease. Nat. Rev. Neurol. 10, 217-224.

Perry, V. H. and Teeling, J. (2013) Microglia and macrophages of the central nervous system: the contribution of microglia priming and systemic inflammation to chronic neurodegeneration. Semin. Immunopathol. 35, 601-612.

Pott Godoy, M. C., Tarelli, R., Ferrari, C. C., Sarchi, M. I. and Pitossi, F. J. (2008) Central and systemic IL-1 exacerbates neurodegeneration and motor symptoms in a model of Parkinson's disease. Brain 
131, 1880-1894.

Przedborski, S. (2017) The two-century journey of Parkinson disease research. Nat. Rev. Neurosci. 18, 251-259.

Seok, J. K., Kang, H. C., Cho, Y. Y., Lee, J. S. and Lee, J. Y. (2021) Therapeutic regulation of the NLRP3 inflammasome in chronic inflammatory diseases. Arch. Pharm. Res. 44, 16-35.

Swanson, K. V., Deng, M. and Ting, J. P. (2019) The NLRP3 inflammasome: molecular activation and regulation to therapeutics. Nat. Rev. Immunol. 19, 477-489.

Wang, W., Nguyen, L. T., Burlak, C., Chegini, F., Guo, F., Chataway, T., Ju, S., Fisher, O. S., Miller, D. W., Datta, D, Wu, F., Wu, C. X., Landeru, A., Wells, J. A., Cookson, M. R., Boxer, M. B., Thomas, C. J., Gai, W. P., Ringe, D., Petsko, G. A. and Hoang, Q. Q. (2016) Caspase- 1 causes truncation and aggregation of the Parkinson's disease-associated protein $\alpha$-synuclein. Proc. Natl. Acad. Sci. U.S.A. 113, 9587-9592.
Wen, A. Y., Sakamoto, K. M. and Miller, L. S. (2010) The role of the transcription factor CREB in immune function. J. Immunol. 185, 6413-6419.

Wilson, L. S. and Brandon, N. J. (2015) Emerging biology of PDE10A. Curr. Pharm. Des. 21, 378-388.

Yan, Y., Jiang, W., Liu, L., Wang, X., Ding, C., Tian, Z. and Zhou, R. (2015) Dopamine controls systemic inflammation through inhibition of NLRP3 inflammasome. Cell 160, 62-73.

Zagorska, A., Partyka, A., Bucki, A., Gawalskax, A., Czopek, A. and Pawlowski, M. (2018) Phosphodiesterase 10 inhibitors - novel perspectives for psychiatric and neurodegenerative drug discovery. Curr. Med. Chem. 25, 3455-3481.

Zhu, W., Liu, S., Zhao, J., Liu, S., Jiang, S., Li, B., Yang, H., Fan, C. and Cui, W. (2014) Highly flexible and rapidly degradable papaverineloaded electrospun fibrous membranes for preventing vasospasm and repairing vascular tissue. Acta Biomater. 10, 3018-3028. 